between the $\mathrm{CD} 4^{+} \mathrm{T}$ cells and DCs (Figure 1), as MHC class II-mismatched DCs, which are unable to present antigen to $\mathrm{T}$ cells, did not induce Th17 differentiation $(10,11)$.

\section{Conclusions}

Given the importance of IL-17 in autoimmune disease, the mechanisms of Th17 differentiation are under extensive study. The works presented by Acharya et al. (10) and Melton et al. (11) demonstrate a novel mechanism for the development of Th17 cells, in which naive $\mathrm{CD} 4^{+} \mathrm{T}$ cells recognize antigens presented by DCs in an MHC class II-dependent manner, while at the same time inducing the cell to differentiate to a Th17 cell through the activation of TGF- $\beta$ by an integrin $\alpha v \beta 8$-dependant mechanism (Figure 1). While these studies do not explain the production of IL-17 by other sources, such as $\gamma \delta$ $\mathrm{T}$ cells, they do offer insight into the development of an important cell lineage that is implicated in autoimmune states. They also suggest the use of RGD mimetics to block the activation of TGF- $\beta$ could be a feasible therapy to reduce the severity of Th17related diseases. Recently, however, work by Ghoreschi et al. demonstrates that Th17 cells can develop in the absence of TGF- $\beta$, and Th17 cells grown in these conditions show enhanced pathogenic potential after adoptive transfer (19). These data highlight the complexities of Th17 differentiation and suggest that it will be important to under- stand the origins and phenotypes of Th17 cells (and their nuanced subsets) in order to develop therapeutic approaches.

\section{Acknowledgments}

The authors would like to acknowledge support from the following Public Health Service grants: 5R01HL079142 (to D.A. Pociask and J.K. Kolls) and P50HL084932 (to J.K. Kolls).

Address correspondence to: Jay K. Kolls, Department of Genetics, Louisiana State University Health Sciences Center, New Orleans, Louisiana 70112, USA. Phone: 504.568.6117; Fax: 504.568.8500; E-mail: jkolls@1suhsc.edu.

1. Weaver CT, Hatton RD, Mangan PR, Harrington LE. IL-17 family cytokines and the expanding diversity of effector T cell lineages. Annu Rev Immunol. 2007;25:821-852

2. Korn T, Bettelli E, Oukka M, Kuchroo VK. IL-17 and Th17 cells. Annu Rev Immunol. 2009;27:485-517.

3. Dong C. TH17 cells in development: an updated view of their molecular identity and genetic programming. Nat Rev Immunol. 2008;8(5):337-348.

4. Khader SA, Gaffen SL, Kolls JK. Th17 cells at the crossroads of innate and adaptive immunity against infectious diseases at the mucosa. Mucosal Immunol. 2009;2(5):403-411.

5. Shevach EM. Mechanisms of foxp $3+\mathrm{T}$ regulatory cell-mediated suppression. Immunity. 2009; 30(5):636-645

6. Bettelli E, et al. Reciprocal developmental pathways for the generation of pathogenic effector TH17 and regulatory T cells. Nature. 2006;441(7090):235-238.

7. Mangan PR, et al. Transforming growth factor- $\beta$ induces development of the TH17 lineage. Nature. 2006;441(7090):231-234.

8. Veldhoen M, Hocking RJ, Atkins CJ, Locksley RM,
Stockinger B. TGFbeta in the context of an inflammatory cytokine milieu supports de novo differentiation of IL-17-producing T cells. Immunity. 2006;24(2):179-189.

9. Li MO, Wan YY, Flavell RA. T cell-produced transforming growth factor-beta1 controls $\mathrm{T}$ cell tolerance and regulates Th1- and Th17-cell differentiation. Immunity. 2007;26(5):579-591.

10. Acharya $M$, et al. $\alpha v$ Integrin expression by DCs is required for Th17 cell differentiation and development of experimental autoimmune encephalomyelitis in mice. J Clin Invest. 2010;120(12):4445-4452.

11. Melton AC, Bailey-Bucktrout SL, Travis MA, Fife BT, Bluestone JA, Sheppard D. Expression of $\alpha v \beta 8$ integrin on dendritic cells regulates Th17 cell development and experimental autoimmune encephalomyelitis in mice. J Clin Invest. 2010;120(12):4436-4444.

12. Mantel PY, Schmidt-Weber CB. Transforming growth factor-beta: recent advances on its role in immune tolerance. Methods Mol Biol. 2011;677:303-338.

13. Annes JP, Munger JS, Rifkin DB. Making sense of latent TGFbeta activation. J Cell Sci. 2003; 116 (pt 2):217-224.

14. Yang $Z$, et al. Absence of integrin-mediated TGFbeta 1 activation in vivo recapitulates the phenotype of TGFbeta1-null mice. J Cell Biol. 2007; 176(6):787-793.

15. Munger JS, et al. The integrin alpha v beta 6 binds and activates latent TGF beta 1: a mechanism for regulating pulmonary inflammation and fibrosis. Cell. 1999;96(3):319-328.

16. Mu D, et al. The integrin alpha(v)beta8 mediates epithelial homeostasis through MT1-MMP-dependent activation of TGF-beta1. J Cell Biol. 2002; 157(3):493-507.

17. Lacy-Hulbert A, et al. Ulcerative colitis and autoimmunity induced by loss of myeloid alphav integrins. Proc Natl Acad Sci U S A. 2007;104(40):15823-15828.

18. Travis MA, et al. Loss of integrin alpha(v)beta 8 on dendritic cells causes autoimmunity and colitis in mice. Nature. 2007;449(7160):361-365.

19. Ghoreschi K, et al. Generation of pathogenic $\mathrm{T}(\mathrm{H}) 17$ cells in the absence of TGF-beta signalling. Nature. 2010;467(7318):967-971.

\title{
A tincture of hepcidin cures all: the potential for hepcidin therapeutics
}

\author{
Thomas B. Bartnikas and Mark D. Fleming
}

Department of Pathology, Children's Hospital Boston, Boston, Massachusetts, USA.

\begin{abstract}
Iron overload as a result of blood transfusions and excessive intestinal iron absorption can be a complication of chronic anemias such as $\beta$-thalassemia. Inappropriately low levels of hepcidin, a negative regulator of iron absorption and recycling, underlie the pathophysiology of the intestinal hyperabsorption. In this issue of the JCI, Gardenghi et al. demonstrate that increasing hepcidin expression to induce iron deficiency in murine $\beta$-thalassemia not only mitigates the iron overload, but also the severity of the anemia. These data illustrate the therapeutic potential of modulating hepcidin expression in diseases associated with altered iron metabolism.
\end{abstract}

Conflict of interest: The authors have declared that no conflict of interest exists.

Citation for this article: $J$ Clin Invest. 2010;

120(12):4187-4190. doi:10.1172/JCI45043.

\section{Hepcidin, iron, and erythropoiesis}

Erythropoiesis consumes the majority of the iron present in the human body (1). Most of this iron is obtained from the recycling of effete red blood cells by macrophages found in the liver, spleen, and bone marrow. Interruption of iron export from macrophages leads to functional iron deficiency and iron-limited erythropoiesis. At equilibrium, only a small amount of iron is absorbed in the duodenum from the diet each day. Further, there is no physiologically regulated mechanism of eliminating excess iron from the body. Consequently, the proper regulation of dietary iron absorption as well as iron recycling is essential to maintaining iron homeostasis and to sustaining erythropoiesis. 


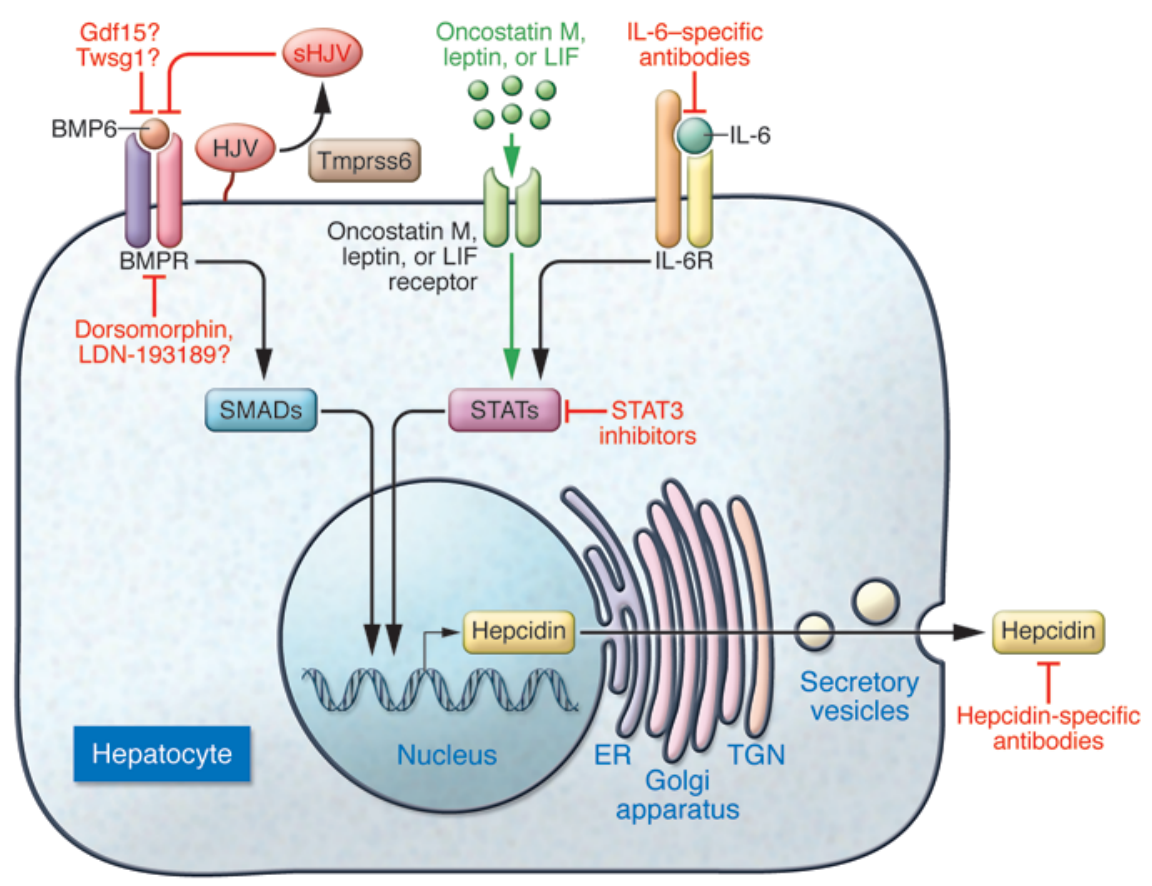

The peptide hormone hepcidin is the key regulator of iron absorption and recycling (2). Secreted predominantly by hepatocytes, hepcidin binds to and induces the internalization and degradation of ferroportin, an iron export protein present on the cell surface of macrophages and duodenal enterocytes. In this manner, hepcidin limits iron recycling and absorption, thereby controlling body iron stores and the availability of iron for erythropoiesis. As a negative regulator, hepcidin expression is inversely correlated with body iron stores. Importantly, hepcidin expression is inhibited by erythropoietic activity through an as-yetunknown mechanism conceptualized as the "erythroid regulator" of iron homeostasis. This signal is particularly strong in individuals with anemia, such as those with $\beta$-thalassemia.

Hemoglobin is composed of two $\alpha$-globin and two $\beta$-globin chains, with each chain having one associated heme moiety. $\beta$-thalassemia is an inherited disorder of $\beta$-globin synthesis due to mutations that result in decreased levels of a structurally normal $\beta$-globin protein. In $\beta$-thalassemia, excess unpaired $\alpha$-globin chains resulting from the $\beta$-globin synthesis defect are toxic to erythroid precursors, causing their premature death in the bone marrow. Such abortive erythropoiesis is termed "ineffective erythropoiesis" and is a particularly potent inducer of the erythroid regulator of iron homeostasis. Chronically low levels of hep- cidin lead to excessive dietary iron absorption and iron overload, which manifests clinically as liver disease, cardiomyopathy, and endocrinopathies. The hepcidin suppression caused by ineffective erythropoiesis is so strong that individuals with $\beta$-thalassemia intermedia, a moderately severe form of $\beta$-thalassemia in which patients do not require frequent blood transfusions to survive, can develop clinically significant iron overload. In severe $\beta$-thalassemia ( $\beta$-thalassemia major) chronic blood transfusions, employed to correct the anemia and suppress ineffective erythropoiesis, lead to massive iron overload associated with high hepcidin levels. In either case, iron overload is a major cause of morbidity and mortality in these patients, necessitating pharmacological iron chelation.

Therein lies the paradox of iron homeostasis and hepcidin in the setting of ineffective erythropoiesis and, in particular, $\beta$-thalassemia intermedia: iron is required to make red blood cells to correct the anemia, and the body responds to ineffective erythropoiesis by decreasing hepcidin levels to provide iron, yet the response is in some manner maladaptive, as it leads to chronic iron overload and its attendant toxicities. If hepcidin levels are inappropriately low for the degree of iron stores in $\beta$-thalassemia intermedia, then increasing them might attenuate the severity of iron overload. In this issue of the JCI, Gardenghi et al. test just that (3).

\section{Figure 1}

Potential ways in which hepcidin expression and activity could be modulated pharmacologically. Shown in a schematized hepatocyte are several pathways leading to hepcidin expression. BMP6 stimulates hepcidin expression in a pathway dependent upon HJV, BMP receptors (BMPRs), and SMAD activation; Tmprss6 cleaves membrane-bound HJV, producing soluble HJV (sHJV). IL-6 stimulates hepcidin synthesis via STAT activation. For simplicity, not all factors involved with hepcidin expression are shown.

\section{The therapeutic potential of hepcidin in $\beta$-thalassemia}

Hypothesizing that $\beta$-thalassemic mice absorb more dietary iron than is needed for erythropoiesis, Gardenghi et al. placed $\beta$-thalassemic mice on a low-iron diet (3). As expected, iron levels significantly decreased. Remarkably, and somewhat unexpectedly, however, erythropoiesis was relatively unaffected and the anemia and splenomegaly actually improved. Next, the authors reasoned that increasing hepcidin expression could have the same effect as limiting dietary iron. To this end, they took several approaches. First, they used viral transduction to overexpress the hemochromatosis gene $\mathrm{Hfe}$, which encodes a membrane protein that promotes hepcidin expression, in the livers of $\beta$-thalassemic mice and demonstrated a decrease in tissue iron content without any adverse effects on erythropoiesis. Second, they bred the anemic animals to a transgenic line that constitutively expresses low levels of hepcidin. $\beta$-thalassemic mice carrying the transgene demonstrated an improvement in their anemia and decreased tissue iron content at one and five months of age.

A major determinant of the pathophysiology of $\beta$-thalassemia is the accumulation of precipitated unpaired $\alpha$-globin chains and membrane damage caused by partially denatured, oxidized hemoglobin derivatives such as hemichrome. In the hepcidin-expressing transgenic mice, 
Gardenghi et al. found lower levels of membrane-associated $\alpha$-globin chains as well as decreased red blood cell reactive oxygen species (3), compatible with the notion that slightly increased hepcidin levels ultimately relieved the proximal cause of cell damage and death. Whether the observed improvement in the anemia was due to less ineffective erythropoiesis, increased red blood cell lifespan, or a combination thereof, remains to be determined. However, it seems likely that the effect may be a consequence of a reduced rate of $\alpha$-compared with $\beta$-globin synthesis induced by iron deficiency (4). Intriguingly, several mice expressing very high levels of hepcidin had more severe anemia, indicating that there is very likely a narrow therapeutic window of hepcidin efficacy in this disease model.

The paper by Gardenghi et al. (3) is timely in that it complements a recent publication by Li et al. (5). In that paper, the authors demonstrated that serial injections of transferrin, the iron-binding protein in the serum that is essential for iron delivery to erythroid progenitors, leads to increased hepcidin expression and significant improvements in anemia in the same mouse model of $\beta$-thalassemia. While Li et al. explained their findings by concluding that transferrin is a limiting factor in $\beta$-thalassemia (5), the work of Gardenghi et al. suggests that downstream effects on hepcidin or restricted erythroid iron delivery could be the major contributing factor (3).

\section{Hepcidin-targeted therapeutics in other iron metabolism disorders}

Altering hepcidin levels and/or activity has also proven beneficial in disorders other than $\beta$-thalassemia (Figure 1). Transgenic overexpression of hepcidin and injections of synthetic hepcidin both lead to reduced tissue iron levels in mouse models of genetic hemochromatosis, a group of diseases of hepcidin deficiency and iron overload generally due to mutations in factors required for hepcidin expression $(6,7)$. Modulation of hepcidin activity and/or levels may also be of clinical utility in inflammatory disorders associated with anemia (i.e., the anemia of inflammation), in which elevated levels of proinflammatory cytokines such as IL-6 lead to hepcidin overexpression and promote iron-restricted erythropoiesis. Indeed, administration of hepcidin-specific antibodies increases serum iron levels in a mouse model of the anemia of inflammation (8). In addition, administration of IL-6-specific antibodies reduces hepcidin levels and improves anemia in a monkey collagen-induced arthritis model (9) as well as reduces serum hepcidin levels in human patients with Castleman disease, a lymphoproliferative disorder associated with high levels of IL-6 (10). Targeting downstream mediators of inflammation-induced hepcidin expression such as the JAK/STAT signaling pathway may also prove efficacious in the anemia of inflammation. While STAT3 inhibitors inhibit hepcidin expression in vitro, the cytokine oncostatin $M$, the hormone leptin, and the cytokine leukemia inhibitory factor (LIF) all activate the STAT pathway in vitro; oncostatin M has also been shown to increase hepcidin expression in mice (11-14).

Hepcidin levels can also be altered by targeting a bone morphogenetic proteindependent (BMP-dependent) pathway known to be central to regulation of hepcidin expression. This pathway involves a multitude of extracellular, membranebound, and intracellular factors (2). Growth differentiation factor 15 (Gdf15) and twisted gastrulation homolog 1 (Twsg1), both BMP antagonists produced by erythroblasts and putative components of the erythroid regulator, inhibit hepcidin expression in vitro, although their physiologic relevance has not been established definitively (15). Administration of dorsomorphin, a BMP type I receptor inhibitor, decreases hepcidin expression in mice (16). While dorsomorphin and its more receptor-selective derivative compound LDN-193189 have not been tested in humans, these agents represent an exciting potential therapy for a variety of disorders associated with inappropriately high hepcidin levels, such as the anemia of inflammation. Likewise, the hepatocyte BMP receptor complex and signaling cascade might equally be therapeutic targets in conditions associated with hepcidin excess. For example, hemojuvelin (HJV; also known as HFE2), which is mutated in a severe form of juvenile onset hemochromatosis, is required for hepcidin expression and believed to act as a BMP coreceptor (2). Its levels are regulated by the activity of two other proteins, transmembrane protease serine 6 (Tmprss6) and neogenin, which are themselves potential drug targets (17). Mutations in Tmprss 6 cause iron-responsive iron deficiency anemia (IRIDA), a form of inherited microcytic anemia due to hepcidin overexpression, and lead to excessive hepcidin production in humans and mice, while neogenin-deficient mice exhibit hepcidin deficiency and iron overload. Tmprss6 cleaves HJV from the cell surface, thereby liberating a soluble form of HJV, while neogenin inhibits secretion of HJV from hepatocytes. Soluble HJV inhibits hepcidin expression in vitro, but this agent has yet to be tested in vivo (18). Finally, liver-specific deletion of the downstream transcription factor SMAD4 leads to hepcidin deficiency and iron overload in mice, suggesting that antagonism of SMAD4 activity could downregulate hepcidin expression (19).

\section{Hope for the future}

Overall, the work of Gardenghi et al. (3) is exciting in that it represents a proof of concept that diseases associated with iron overload can be treated by modulating hepcidin expression and/or activity. Furthermore, their data suggest that manipulating this pathway may be a method of not only treating the secondary iron overload associated with $\beta$-thalassemia intermedia, but also of ameliorating the anemia itself. Because of its short half-life, pharmacologic means of increasing the expression of endogenous hepcidin and/or its downstream activity, rather than administering hepcidin itself, would be the ideal therapeutic. Furthermore, in practical terms, a multipronged approach involving hepcidin modulators and iron chelation might be the most effective strategy. Whatever the eventual method might be, it is clear that hepcidin is now a well-validated target earnestly awaiting human application.

\section{Acknowledgments}

The authors thank David G. Nathan and H. Franklin Bunn for helpful discussions. M.D. Fleming is supported by NIH R01 DK080011. T.B. Bartnikas is supported by NIH K99 DK084122.

Address correspondence to: Mark D. Fleming, Enders 1116.1, Children's Hospital, 300 Longwood Ave., Boston, Massachusetts 02115, USA. Phone: 617.919.2664; Fax:617.730.0168; E-mail: mark.fleming@ childrens.harvard.edu.

\footnotetext{
1. Andrews NC. Disorders of iron metabolism. N Engl IMed. 1999;341(26):1986-1995.

2. Fleming MD. The regulation of hepcidin and its effects on systemic and cellular iron metabolism. Hematology Am Soc Hematol Educ Program. 2008:151-158.

3. Gardenghi S, et al. Hepcidin as a therapeutic tool to limit iron overload and improve anemia in $\beta$-thalassemic mice. J Clin Invest. 2010;120(12):4466-4477.

4. Ben-Bassat I, Mozel M, Ramot B. Globin synthesis in iron-deficiency anemia. Blood. 1974;44(4):551-555.

5. Li H, et al. Transferrin therapy ameliorates disease in beta-thalassemic mice. Nat Med. 2010;16(2):177-182.
} 
6. Nicolas G, et al. Constitutive hepcidin expression prevents iron overload in a mouse model of hemochromatosis. Nat Genet. 2003;34(1):97-101.

7. Morán-Jiménez M, et al. Hepcidin treatment in Hfe -/- mice diminishes plasma iron without affecting erythropoiesis. EurJ Clin Invest. 2010;40(6):511-517.

8. Sasu BJ, et al. Antihepcidin antibody treatment modulates iron metabolism and is effective in a mouse model of inflammation-induced anemia. Blood. 2010;115(17):3616-3624.

9. Hashizume M, Uchiyama Y, Horai N, Tomosugi N, Mihara M. Tocilizumab, a humanized anti-interleukin- 6 receptor antibody, improved anemia in monkey arthritis by suppressing IL-6-induced hepcidin production. Rheumatol Int. 2010;30(7):917-923.

10. Song SJ, Tomosugi N, Kawabata H, Ishikawa T, Nishikawa T, Yoshizaki K. 2010. Downregulation of hepcidin resulting from long-term treatment with an anti-IL-6 receptor antibody (tocilizumab) improves anemia of inflammation in multicentric Castleman's disease (MCD) [published online ahead of print July 19, 2010]. Blood. doi: 10.1182/ blood-2010-03-271791.

11. Fatih N, et al. Natural and synthetic STAT3 inhibitors reduce hepcidin expression in differentiated mouse hepatocytes expressing the active phosphorylated STAT3 form. J Mol Med. 2010;88(5):477-486.

12. Chung B, Matak P, McKie AT, Sharp P. Leptin increases the expression of the iron regulatory hormone hepcidin in HuH7 human hepatoma cells. J Nutr. 2007;137(11):2366-2370.

13. Kanda J, Uchiyama T, Tomosugi N, Higuchi M, Uchiyama T, Kawabata H. Oncostatin M and leukemia inhibitory factor increase hepcidin expression in hepatoma cell lines. Int J Hematol. 2009;90(5):545-552.

14. Chung B, Verdier F, Matak P, Deschemin J, Mayeux P, Vaulont $\mathrm{S}$. Oncostatin $\mathrm{M}$ is a potent inducer of hepcidin, the iron regulatory hormone. FASEB J. 2010;
24(6):2093-2103

15. Tanno T, Miller JL. Iron loading and overloading due to ineffective erythropoiesis. Adv Hematol. 2010; 2010:358283

16. Yu PB, et al. Dorsomorphin inhibits BMP signals required for embryogenesis and iron metabolism. Nat Chem Biol. 2008;4(1):33-41.

17. Lee D, et al. Neogenin inhibits HJV secretion and regulates BMP-induced hepcidin expression and iron homeostasis. Blood. 2010;115(15):3136-3145.

18. Nili M, Shinde U, Rotwein P. Soluble RGMc/hemojuvelin is a broad spectrum BMP antagonist and inhibits both BMP2- and BMP6-mediated signaling and gene expression [published online ahead of print June 8, 2010]. J Biol Chem. doi: 10.1074/jbc. M110.130286.

19. Wang $\mathrm{R}$, et al. A role of SMAD4 in iron metabolism through the positive regulation of hepcidin expression. Cell Metab. 2005;2(6):399-409.

\title{
Can TNF- $\alpha$ boost regulatory T cells?
}

\author{
Angelina M. Bilate and Juan J. Lafaille
}

\begin{abstract}
Molecular Pathogenesis Program, Kimmel Center for Biology and Medicine at the Skirball Institute Department of Pathology, New York University School of Medicine, New York, New York, USA.
\end{abstract}

\begin{abstract}
Deleterious immune responses that cause autoimmune diseases such as type 1 diabetes are normally kept in check by a myriad of mechanisms. Among these, protection mediated by $\mathrm{CD}^{+} \mathrm{Foxp}^{+}$Tregs constitutes an essential pathway. Much work over the past decade aimed to understand how Tregs affect immune responses triggered by effector $T$ cells (Teffs), but less is known about how Teffs affect Tregs. In this issue of the JCI, Grinberg-Bleyer et al. report the clearest example thus far regarding this important aspect of Treg biology. They find that in mice, sustained protection from diabetes by Tregs is dependent on Teffs and partially dependent on TNF- $\alpha$, a cytokine traditionally considered proinflammatory.
\end{abstract}

Chronic inflammatory diseases such as asthma and autoimmune diseases such as type 1 diabetes arise from the breakdown of the mechanisms that normally restrain immune responses. Key among those mechanisms is a subset of $\mathrm{CD} 4^{+} \mathrm{T}$ cells called Tregs. Tregs are characterized by expression of the transcription factor forkhead box P3 (Foxp3). Foxp3 is not only important for the development and maintenance of Tregs but also for their suppressive function $(1,2)$. Perhaps the best evidence for the indispensable role of Tregs in preventing autoimmunity and limiting chronic inflammatory diseases comes from the fact that defective development of Tregs in humans with FOXP3 mutations leads to the life-threatening autoimmune condition immune dysregu-

Conflict of interest: The authors have declared that no conflict of interest exists.

Citation for this article: J Clin Invest. 2010; 120(12):4190-4192. doi:10.1172/JCI45262. lation, polyendocrinopathy, enteropathy, and X-linked (IPEX) syndrome $(3,4)$. A similar lethal disease is observed in scurfy mice, which lack Tregs due to mutations in the Foxp3 gene (5). In this issue of the JCI, Grinberg-Bleyer et al. (6) provide new insight into how mouse $\mathrm{CD} 4^{+} \mathrm{Foxp}^{+}{ }^{+}$Treg numbers and suppressive activities are regulated in the autoimmune setting of type 1 diabetes. Their data support the hypothesis that the very cells that the Tregs are suppressing (diabetogenic effector $\mathrm{T}$ cells [Teffs]) themselves act in a feedback loop to help islet-specific Tregs, providing sustained protection from diabetes, a hypothesis with far-reaching implications.

\section{Tregs depend on Teffs}

The vast majority of studies on Tregs have focused on the mechanisms by which they affect the responses mediated by Teffs. However, it has been noted for some time that there is substantial bidirectionality in the interactions between the two cell populations. As Tregs require IL-2 for their survival and function but do not produce it, it was thought that Teffs would be important providers of IL-2 to Tregs. In vivo mixing experiments with IL-2-deficient and-sufficient Tregs and Teffs confirmed this IL-2-based interdependence of Tregs and Teffs and led to the suggestion that Teffs were required to help maintain a functional Treg compartment $(7,8)$. Other studies provided additional support for the existence of a feedback loop between Tregs and Teffs, a loop that is important for preventing autoimmune and lymphoproliferative disease (9-11). Thus, it has been established that there is interplay between Tregs and Teffs, and, at least in some cases, this interplay has been shown to be mediated by IL-2 produced by the Teffs.

\section{A feedback loop between Tregs and Teffs in type 1 diabetes}

Despite the precedents in the literature (7-11), few reports of the influence of Teffs on Tregs are as clear and informative as the one presented by Grinberg-Bleyer et al. in this issue of the JCI (6). In their study, the authors investigated the effect of Teffs on Tregs using mouse models of autoimmune diabetes.

The authors initially found that, in vivo, Tregs proliferated significantly more when coinjected into mice with activated $T$ cells, both in pancreata and draining pancreatic LNs (PLNs) (6). These results led to the con- 